\title{
Evaluation of Surface Modified Live Biotherapeutic Products for Oral Delivery
}

\author{
Ava M. Vargason and Aaron C. Anselmo*
}

\begin{abstract}
Live biotherapeutic products (LBPs), including symbiotic and genetically engineered bacteria, are a promising class of emerging therapeutics that are widely investigated both preclinically and clinically for their oral delivery to the gastrointestinal (GI) tract. One emergent delivery strategy involves the direct functionalization of LBP surfaces through noncovalent or covalent modifications to control LBP interactions with the GI microenvironment, thereby improving their viability, attachment, or therapeutic effect. However, unlike other therapeutic modalities, LBPs are living organisms which present two unique challenges for surface modifications: (1) this approach can directly interfere with key LBP biological processes (e.g., colonization, metabolite secretion) and (2) modification can be variable due to the dynamic nature of LBP surfaces. Collectively, these factors remain uncharacterized as they relate to the oral

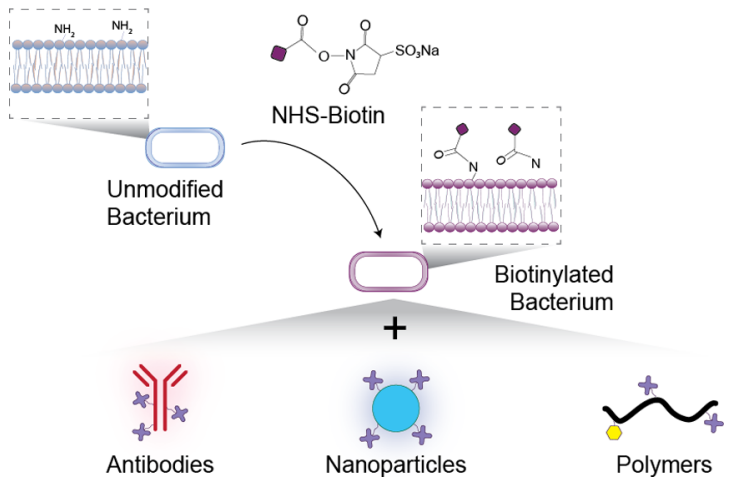
delivery of LBPs. Herein, we leverage our previously reported surface modification platform, which enables LBP surface-presentation of targeting ligands, to broadly evaluate and characterize surface modifications on LBPs. Specifically, we evaluate how LBP growth affects the dilution of surface-presented targeting ligands and the subsequent loss of specific target attachment over time. Next, we describe key surface modification parameters (e.g., concentration, residence time) that can be optimized to facilitate LBP target attachment. We then characterize how bioconjugation influences the suitability of LBPs for oral delivery by evaluating their growth, viability, storage, toxicity against mammalian cells, and in vivo colonization. Broadly, we describe key parameters that influence the performance of surface modified LBPs and subsequently outline an experimental pipeline for characterizing and evaluating their suitability for oral delivery.
\end{abstract}

KEYWORDS: probiotic, drug delivery, bioconjugation, targeted delivery, microbiome

\section{INTRODUCTION}

The human microbiome, consisting of bacteria, fungi, and viruses, plays key roles in metabolizing nutritional sources, maturing the immune system, and preventing pathogen infections. $^{1-3}$ However, therapeutic interventions of the microbiome are largely limited to broad spectrum antibiotics that indiscriminately eliminate commensal bacteria and can induce antibiotic-resistance in pathogens. ${ }^{4,5}$ As such, the use of live biotherapeutic products (LBPs) has emerged as a potential alternative to antibiotic treatment, ${ }^{6}$ as these beneficial bacteria can modulate the composition of the gastrointestinal (GI) microbiome without eliminating commensal species and can be engineered to act as metabolic or biologic-secreting factories. $^{7,8}$

To be effective, LBPs must remain viable under harsh gastric conditions and be retained in the GI tract following oral delivery. ${ }^{9,10}$ As such, a number of strategies have been reported that modify the surface of LBPs to control their interactions with their local microenvironment, ranging from electrostatic absorption, ${ }^{11-15}$ bioconjugation and covalent bonding, ${ }^{16}$ membrane fusing via mechanical extrusion, ${ }^{17}$ antibody- mediated modification, ${ }^{18}$ hydrogen bonding, ${ }^{19}$ and alteration of bacterial growth conditions, ${ }^{20}$ to genetic engineering. ${ }^{21-24}$ These strategies have been employed to reduce immune recognition of administered LBPs, ${ }^{17}$ improve LBP targeting to sites of disease, ${ }^{12,21,23}$ facilitate LBP mucoadhesion, ${ }^{16,24}$ protect LBPs against microenvironment chemical challenges (e.g., acid) in the GI tract, ${ }^{1-15,20}$ and increase LBP therapeutic efficacy. ${ }^{18,22}$ We have recently shown that LBP surfaces can be modified with biotin via $N$-hydroxysulfosuccinimide (NHS) ester-based chemistry, which enables the use of biotin as a modular handle for attachment of streptavidinconjugated moieties (Figure 1). By biotinylating and subsequently decorating LBPs with antibodies, we significantly improved their in vitro attachment and in vivo colonization

Special Issue: Biomaterials for Oral Medicine

Received: September 24, 2020

Accepted: December 18, 2020 

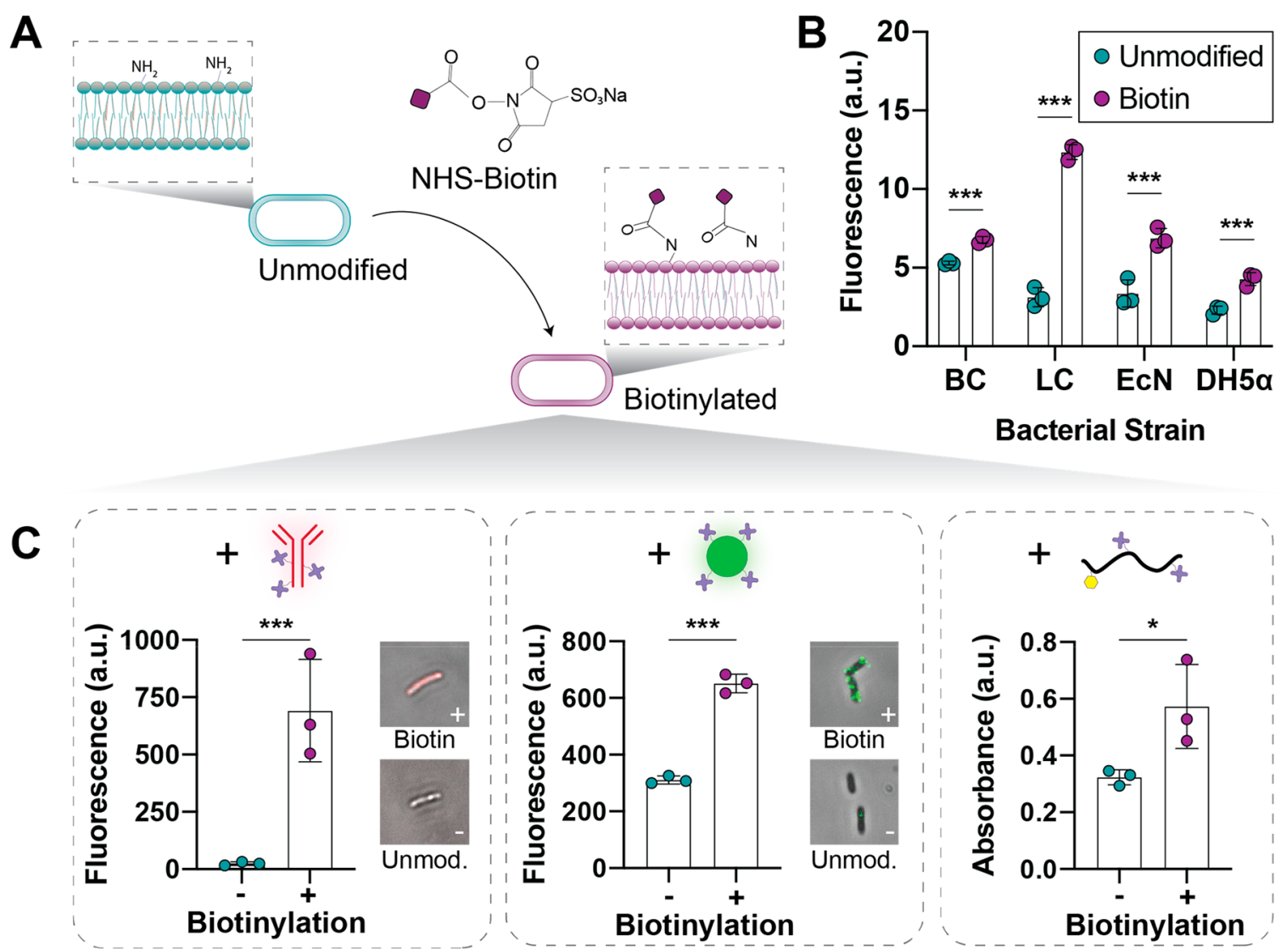

Figure 1. Confirmation of LBP surface biotinylation using NHS-ester chemistry. (A) Schematic of LBP surface biotinylation, where primary amines on the membrane react with $N$-hydroxysulfosuccinimide, forming amide bonds between the LBP surface and biotin. (B) Binding of a fluorescent streptavidin probe by unmodified (green) or biotinylated (pink) Bacillus coagulans (BC), Lactobacillus casei (LC), Escherichia coli Nissle (EcN), and E. coli DH5 $\alpha$ (DHS $\alpha)$, quantified on a microplate reader and (C) the attachment of various streptavidin-conjugated moieties to the surface of DH5 $\alpha$, including an Alexa Fluor 647 IgG antibody (left), yellow-green fluorescent $40 \mathrm{~nm}$ particle (middle), and a horseradish peroxidase tagged polymer (right) ( $n=3$, error shown as standard deviation, significance assessed using unpaired Student's $t$ tests, $\alpha=0.05)$.

following oral delivery. ${ }^{16}$ While surface modifications are a promising delivery strategy for LBPs, these therapeutics are live organisms whose growth, viability, and metabolism can both influence and be influenced by surface modifications. ${ }^{19,25,26}$ As such, there is a need for rigorous characterization of both the effect of the living therapeutic on the surface modification (e.g., ligand dilution as a result of LBP growth), as well as the effect of the surface modification on the living therapeutic (e.g., LBP viability loss as a result of modification).

In this work, we leverage our bioconjugation platform to study how surface modifications affect key LBP functions and how the unique and dynamically changing LBP exterior affects the surface modifications. In doing so, we establish an experimental workflow to characterize surface-modified LBP delivery systems for their oral delivery. This biotinylation platform has a number of advantages that make it ideal as a model system for LBP surface modifications, including a rapid reaction time ( $<20 \mathrm{~min})$, compatibility with any streptavidinconjugated entity or targeting ligand, and modularity across bacterial species due to the use of ubiquitous primary amines for the bioconjugation reaction. ${ }^{27,28}$ Using this previously reported platform, we probe the effects of LBP concentration, residence time, and growth on the attachment of biotinylated LBPs to target proteins. Additionally, we consider parameters related to the clinical translation of LBPs for oral delivery, showing that surface modification does not interfere with therapeutically essential LBP functions, including their ability to survive and grow during standard batch culture, metabolize key therapeutic molecules, and colonize the murine GI tract. Finally, we demonstrate that target binding is conserved for up to 5 months following storage in clinically relevant conditions. Collectively, this work supports the use of LBP bioconjugation as a delivery strategy, while simultaneously establishing an experimental pipeline for the characterization of LBP delivery systems for oral delivery.

\section{RESULTS AND DISCUSSION}

2.1. Biotinylation of LBP Surface. We first demonstrated the modularity of surface modifications across LBP species. Escherichia coli Nissle 1917 (EcN), E. coli DH5 $\alpha$, Lactobacillus casei, and Bacillus coagulans were modified using sulfo-NHSbased chemistry. Specifically, we conjugated biotin to the bacterial surface, which can modulate the attachment of LBPs to biotin's binding partner, streptavidin. Sulfo-NHS-biotin reacts with amine groups on the surface of LBPs, forming an amide bond between the LBP surface and biotin (Figure 1A). To confirm the functional presentation of biotin and altered attachment of streptavidin, we incubated unmodified and biotinylated LBPs with a fluorescent streptavidin probe. Following incubation, the fluorescent signal significantly increased for all strains tested (Figure 1B), and biotinylated LBPs were visibly fluorescent under fluorescence microscopy (Figure S1), confirming the binding of streptavidin to 
A
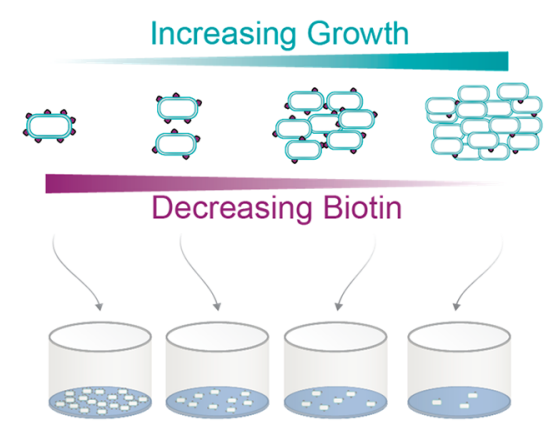

Decreasing Attachment

C

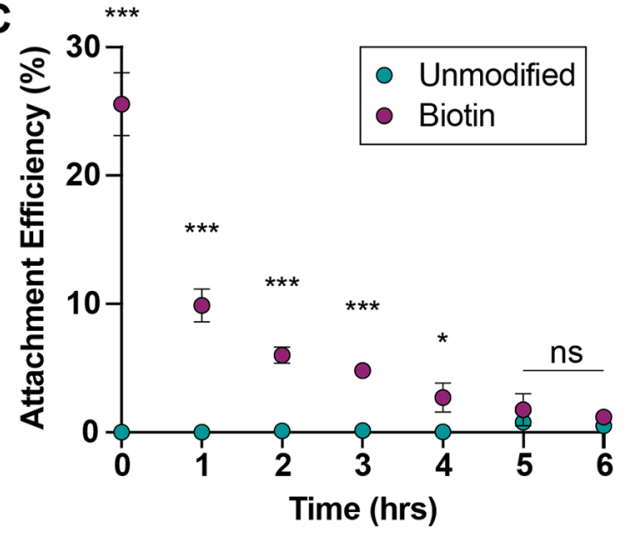

B
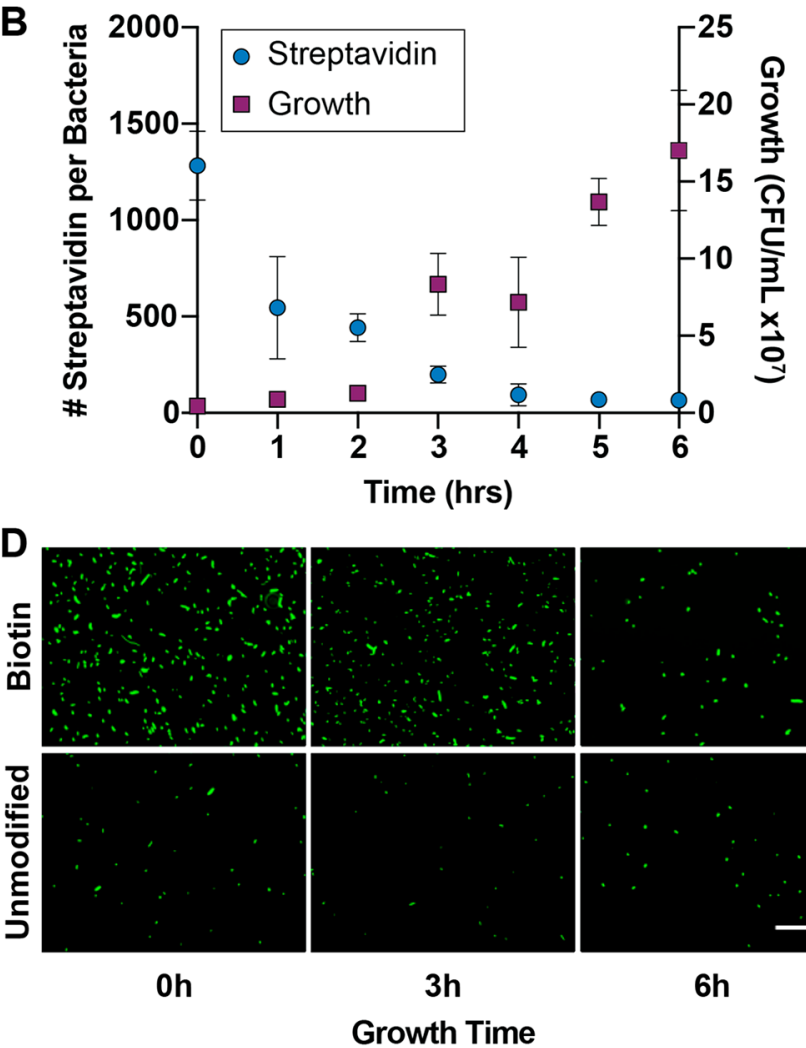

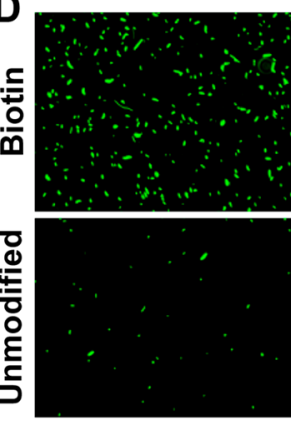

Oh

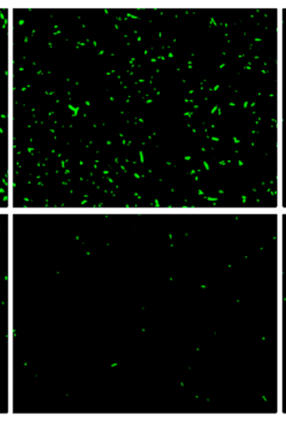

$3 \mathrm{~h}$

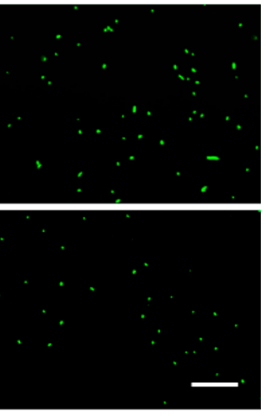

$6 h$

Figure 2. Extent of biotinylation and LBP attachment decreases during growth. (A) The growth and corresponding biotin coverage of modified $E$. coli DH5 $\alpha$ were determined at varying time points (top), and attachment to a streptavidin-coated well-plate was assessed at each time point relative to an unmodified control (bottom). (B) Biotin concentration on the LBP surface (blue circles) at various timepoints during growth (pink squares), measured using a fluorescent streptavidin probe and normalized per colony forming unit (CFU) of bacteria. (C) Attachment efficiency of biotinylated or unmodified LBPs after indicated time points of growth (Attachment Efficiency $=$ Fluorescent Signal Post-Wash $_{\text {Fluorescent }}$ Signal $\left._{\text {Pre-Wash }} * 100\right)$. (D) Representative images of the attached biotinylated (top) and unmodified (bottom) LBPs on the well-plate floor $(n=3$, error shown as standard deviation, significance assessed using two-way ANOVA with Sidak's post hoc test for multiple comparisons, $\alpha=0.05$, $* * *$ $p<0.001, * p<0.05, \mathrm{~ns}=$ not significant). Scale bar $=65 \mu \mathrm{m}$.

biotinylated LBPs. The extent of biotinylation, observed by the intensity of the fluorescent signal, differed between strains (Figure 1B), which is consistent with the abundance of primary amines on these LBP strains (Figure S1) as well as previous reports of this system. ${ }^{16}$ In this platform, biotin acts both as a modulator of bacterial attachment to streptavidin, as well as a modular handle for further modification of the LBP surface (Figure 1C). LBPs can therefore be modified for diverse functionality by altering the moiety on the LBP surface. For example, polymers could reduce the immunogenicity of antigens on the LBP surface, ${ }^{29,30}$ therapeutic nanoparticles conjugated to LBPs can expand their mechanism of action, ${ }^{31}$ and antibodies can improve the attachment and colonization of LBPs in the GI tract following oral delivery. ${ }^{16}$ To demonstrate the attachment of secondary moieties, we modified a fluorescent strain of $E$. coli $D H 5 \alpha$, which enabled us to confirm the identity of the LBP using fluorescence microscopy. LBPs were modified with a streptavidin-conjugated antibody, polymer, and nanoparticle (Figure 1C), detected via fluorescence (antibodies and nanoparticle) or colorimetric (horseradish-peroxidase conjugated polymer) readouts. A GFP-expressing strain was used to confirm antibody attachment, while an RFP-expressing strain was used to confirm nanoparticle attachment to prevent interference between the LBP strain and fluorescent moiety.
2.2. Effects of LBP Parameters on Attachment. A. Growth and Dilution of Surface Modifications. Recently, we showed that surface modification can increase LBP attachment to specific protein targets, including those on abiotic and biotic surfaces. ${ }^{16}$ However, because this platform relies on chemical conjugation, the surface concentration of the conjugated moiety will dilute as LBPs proliferate, potentially impacting attachment due to a reduction in the interactions between the conjugated moiety and its target. While this transient nature of the surface modification ensures the LBP is reverted to its initial form, lowering its safety risk relative to permanent genetic alterations, ${ }^{32,33}$ the dynamics of biotin loss can render insufficient target binding. To better understand the kinetics of this dilution, we chose to analyze a GFP-expressing strain of E. coli DH5 $\alpha$, as its strong GFP signal enables reliable quantification and visualization of LBP attachment. As shown in Figure 2A, we quantified the loss of biotin on the LBP surface during growth and its effect on the attachment of LBPs to a streptavidin-coated well plate. Biotin concentration on the LBP surface was measured using a fluorescent streptavidin probe and approximated by a calibration curve of the probe (Figure S2), normalized to the number of colony forming units $(\mathrm{CFU})$ in a sample. We found that the dilution of surface biotin correlates with the exponential growth of the modified LBP (Figure 2B). To evaluate how dilution of surface moieties affects target attachment, we incubated unmodified and 
A
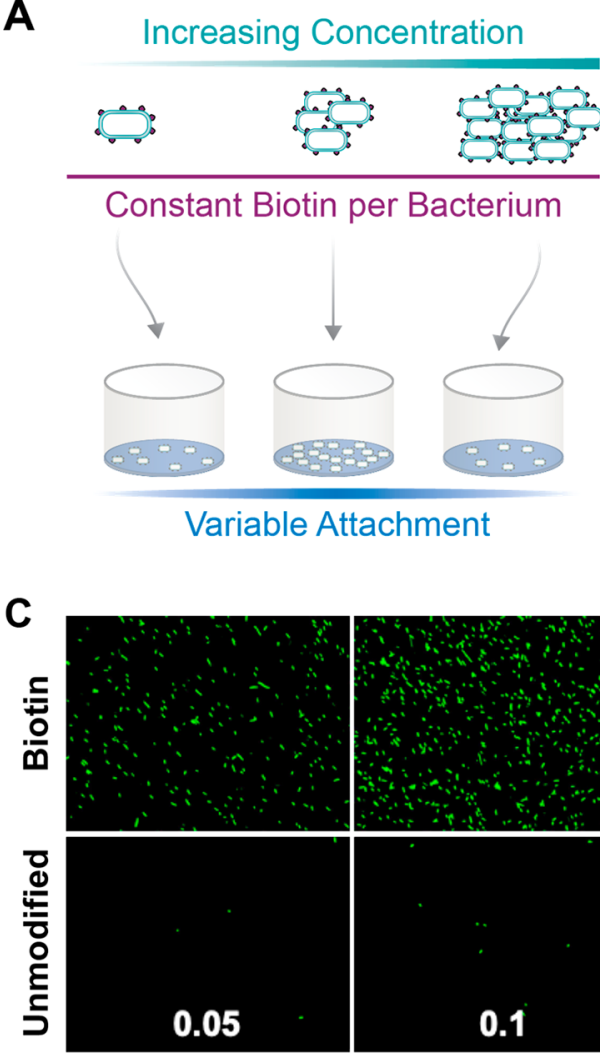
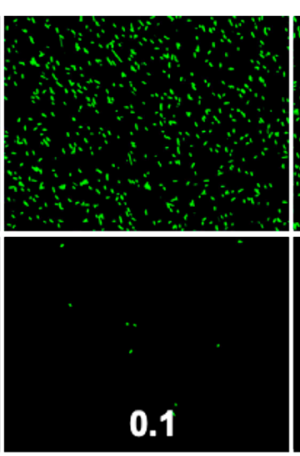
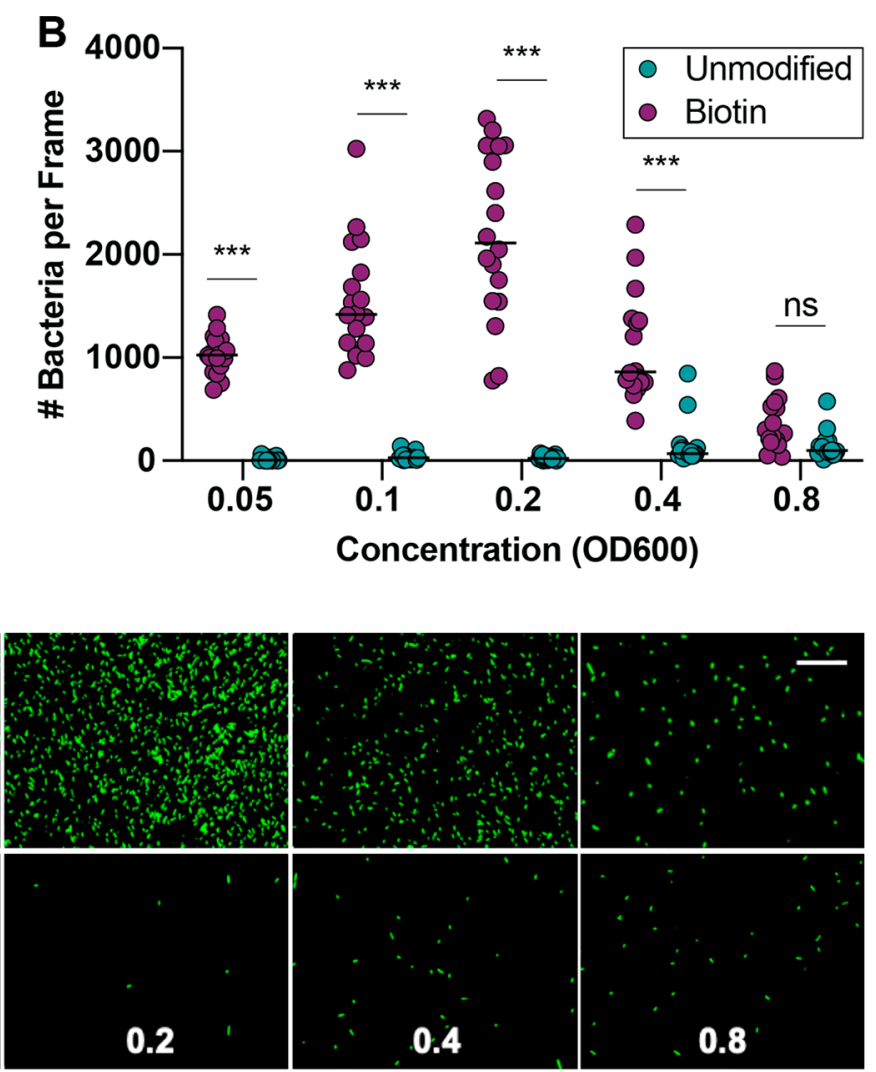

Figure 3. Attachment of modified LBPs is inhibited at high concentrations. (A) E. coli DH5 $\alpha$ was biotinylated and then diluted to varying concentrations. These biotinylated samples or a corresponding unmodified control at the same concentration was incubated on a streptavidincoated well-plate for $20 \mathrm{~min}$. Attachment was assessed using fluorescence intensity and images of the well-plate floor after washing. (B) Attachment of biotinylated (pink) and unmodified (green) LBPs at varying concentrations. Images were quantified using Image $(N=18$, with 3 images per well and 6 wells per condition). (C) Representative images of biotinylated (top) and unmodified (bottom) LBPs (bars represent median, significance assessed using two-way ANOVA with Sidak's post hoc test for multiple comparisons, $\alpha=0.05$, *** $p<0.001$, ns $=$ not significant). Scale bar $=65 \mu \mathrm{m}$.

biotinylated LBPs on a streptavidin-coated plate for $20 \mathrm{~min}$ and calculated the attachment efficiency, measured as the percent of fluorescent signal retained after washing to remove unbound LBPs, at each time point. We found that attachment decreases as a consequence of biotin dilution (Figure 2C), which is supported by fluorescence microscopy of the wellplate floor (Figure 2D). Additionally, we found that the concentration of biotin on the LBP surface begins at $\sim 1300$ biotin molecules per LBP, decreasing to $\sim 550$ after $1 \mathrm{~h}$ of growth and $\sim 70$ after the full $6 \mathrm{~h}$ of growth (Figure $2 \mathrm{~B}$ ). By evaluating the attachment of LBPs at each of these time points during their growth, we discovered that biotinylated LBPs maintain a significantly increased attachment as compared to the unmodified control for up to $4 \mathrm{~h}$ of growth; this corresponds to a minimum concentration of $\sim 100$ biotin molecules per LBP required for enabling enhanced attachment to targeted surfaces.

B. LBP Concentration. We next assessed the effect of LBP concentration on the attachment of biotinylated and unmodified LBPs to target proteins, a key parameter for determining an effective dose following modification. We again chose E. coli DH5 $\alpha$ for these attachment studies, as its fluorescent signal is a reliable and quantifiable indicator of LBP abundance (Figures S3A, S3B, and S4). LBPs were biotinylated as described previously and subsequently diluted to varying concentrations, ensuring the amount of biotin per bacterium $(\sim 1300$, Figure $2 B)$ was identical across all groups. Varying concentrations of biotinylated or unmodified E. coli DH5 $\alpha$ were incubated on a streptavidin-coated well-plate for $20 \mathrm{~min}$ and washed thoroughly (Figure 3A), and LBP attachment was quantified by counting individual, GFP+ attached LBPs in images from the well-plate floor (Figure $3 \mathrm{~B}$ and $3 \mathrm{C}$, Figure $\mathrm{S} 3 \mathrm{C}$ ). It is expected that LBP attachment to an abiotic surface is proportional to concentration, ${ }^{34}$ which we observed in the unmodified control (Figure S5). However, we found that while the attachment of biotinylated LBPs increases with concentration in relatively dilute samples, attachment is inhibited at high concentrations, and a clear attachment maximum exists $(O D=0.2$, Figure $3 B)$. We found that while the specific concentration associated with maximum attachment is dependent on study conditions (Figures S6A and S6B), there is a negative correlation between attachment efficiency and concentration that is conserved across study conditions (Figures S6C and S6D). The inhibition is possibly the result of biotin on the LBP surface becoming sterically hindered as the LBP concentration increases without a corresponding increase to surface area or streptavidin. Therefore, the competition for available streptavidin binding sites will increase and fewer LBPs will reach a sufficient threshold of interactions with their target to maintain attachment following washing.

Next, we analyzed how an initial attachment advantage benefitted biotinylated LBPs during growth. Following attachment in Figure 3B, LBPs were incubated at $37{ }^{\circ} \mathrm{C}$ to allow for growth in $1-\mathrm{h}$ increments and then washed to mimic the 


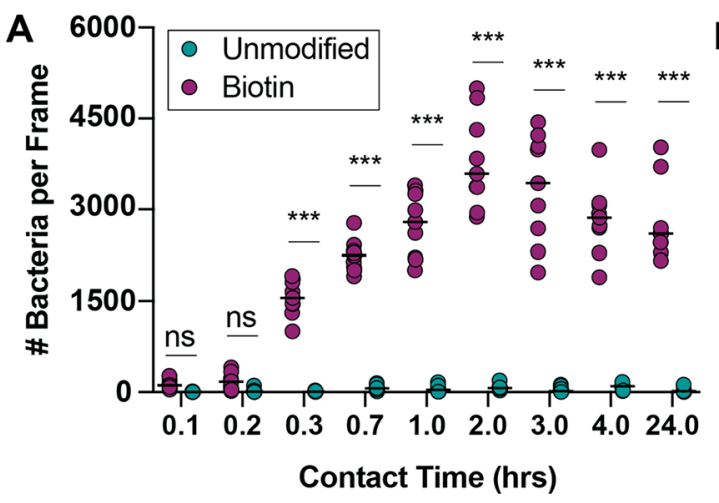

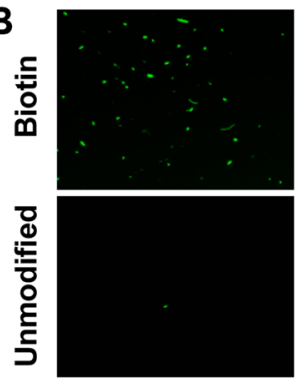

$5 \mathrm{~min}$
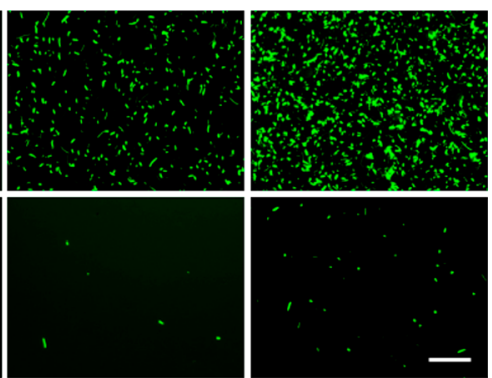

$20 \mathrm{~min}$

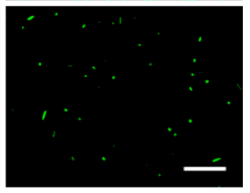

$2 \mathrm{hr}$

Contact Time

Figure 4. Attachment of LBPs increases with residence time. (A) Attachment of unmodified (green) and biotinylated (pink) E. coli DH5 $\alpha$ following incubation on a streptavidin-coated well-plate for indicated time points in PBS at $4{ }^{\circ} \mathrm{C}$. (B) Representative images of the attached biotinylated (top) or unmodified (bottom) LBPs at varying time points (bars represent median, $N=9$, with 3 images per well and 3 wells per condition, significance assessed using two-way ANOVA with Sidak's post hoc test for multiple comparisons, $\alpha=0.05$, *** $p<0.001$, ns $=$ not significant). Scale bar $=65 \mu \mathrm{m}$.
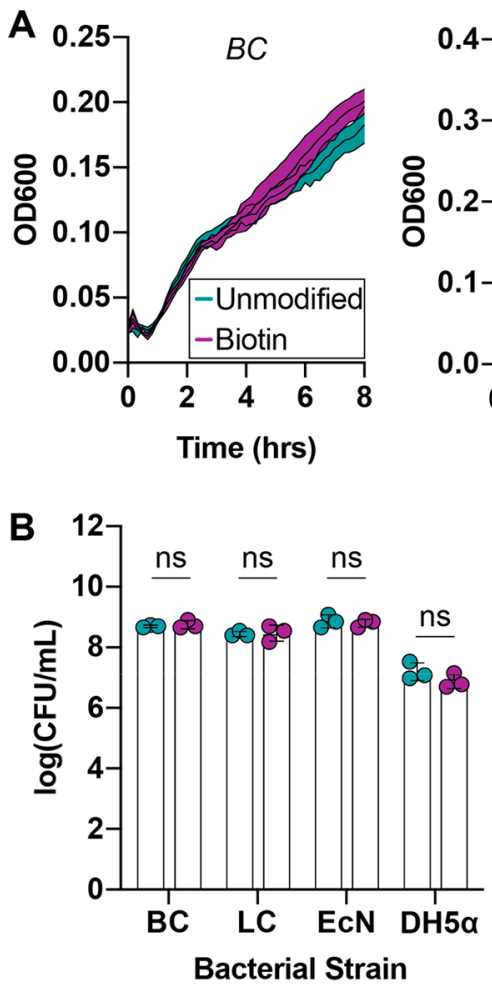
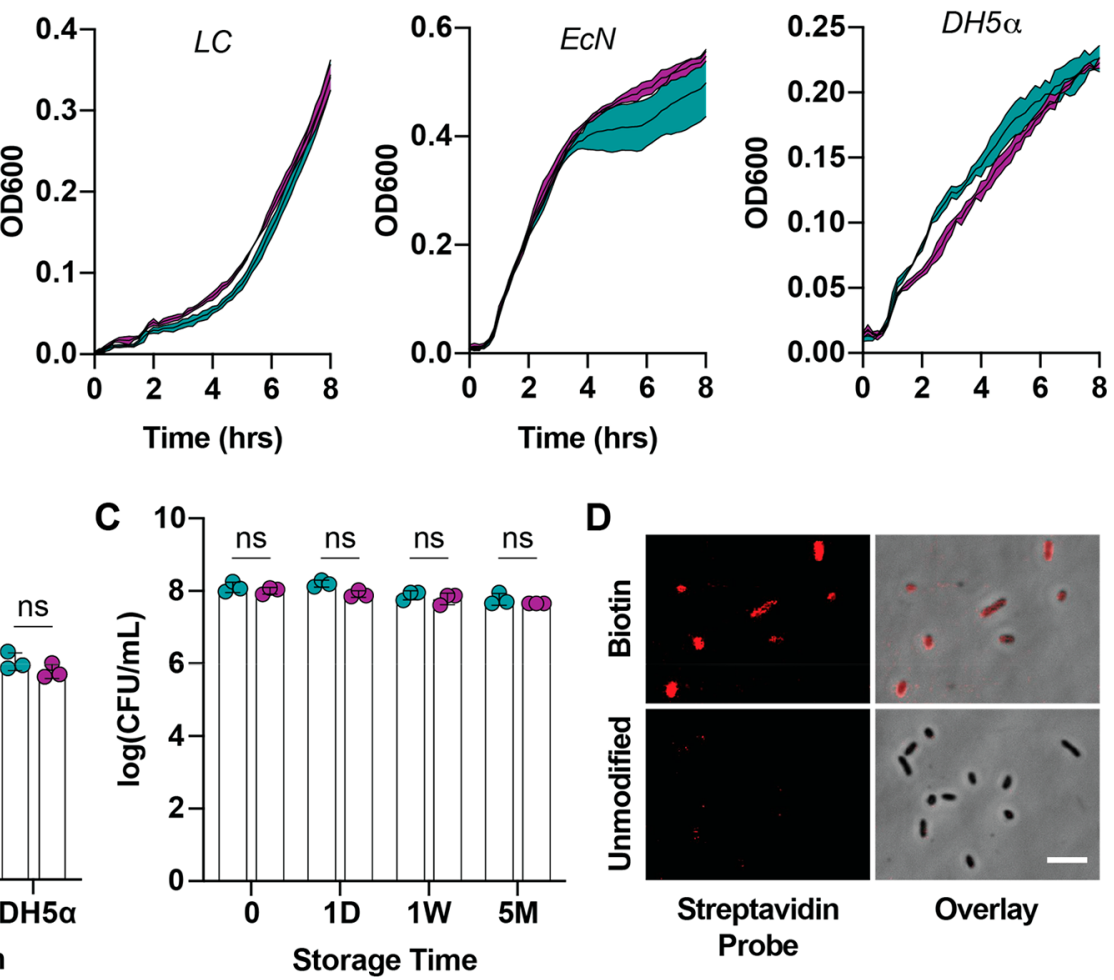

D

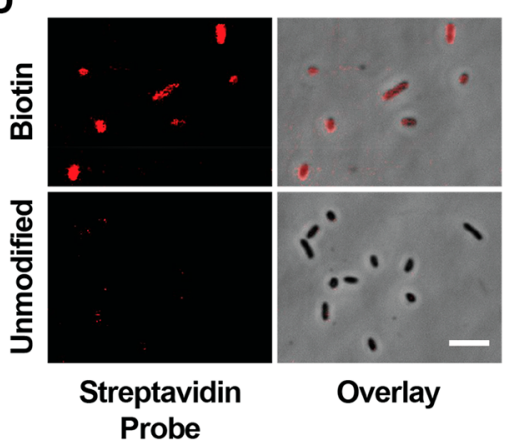

Figure 5. Surface modification is nontoxic and compatible with clinical LBP storage methods. (A) Growth of Bacillus coagulans (BC), Lactobacillus casei (LC), E. coli Nissle 1917 (EcN), and E. coli DH5 $\alpha$ (DH5 $\alpha$ ) before (green) and after biotinylation (pink). (B) LBP viability assessed as colony forming units $(\mathrm{CFU})$ of $\mathrm{BC}, \mathrm{LC}, \mathrm{EcN}$, and $\mathrm{DH} 5 \alpha$ immediately prior to and after biotinylation. (C) Viability of unmodified or biotinylated EcN following storage at $-80^{\circ} \mathrm{C}$ in $25 \%$ glycerol solution prior to storage (0), after 1 day (1D), 1 week (1W), and 5 months (5M) of storage and (D) representative images of biotinylated (top) and unmodified (bottom) $\mathrm{EcN}$ binding a fluorescent streptavidin probe after 5 months of storage ( $n=$ 3 , bars or shading represent standard deviation, significance assessed using multiple unpaired Student's $t$ tests with Holm-Sidak's post hoc test for multiple comparisons in B or two-way ANOVA with Sidak's post hoc test for multiple comparisons in C, ns $=$ not significant). Scale bar $=5 \mu$ m.

dynamic conditions of the GI microenvironment (Figure S7A). We found that the attachment of biotinylated LBPs decreased with time for all concentrations, further supporting that biotin loss on the LBP surface during growth negatively influences attachment, as shown in Figure 2B. Furthermore, biotinylated LBPs retained an attachment advantage over the unmodified control for $5 \mathrm{~h}$ when they are attached prior to growth (Figure S7B). While this study examined a constant concentration of biotin on the LBP surface while varying the concentration of LBP, we next analyzed the effect of varying the sulfo-NHS- biotin reactant concentration with a constant concentration of LBPs. Here, we found that biotin saturates on the surface of the LBP between 0.5 and $1 \mathrm{mg} / \mathrm{mL}$ of sulfo-NHS-biotin in an LBP solution (Figure S8A). Additionally, we found that a clear optimal reactant concentration $(0.25 \mathrm{mg} / \mathrm{mL})$ exists to maximize LBP attachment to a streptavidin-coated well-plate (Figure S8B).

C. Contact Time between LBPs and Target Surface. Finally, we analyzed the effect of residence time on the attachment of modified LBPs to their target protein. 

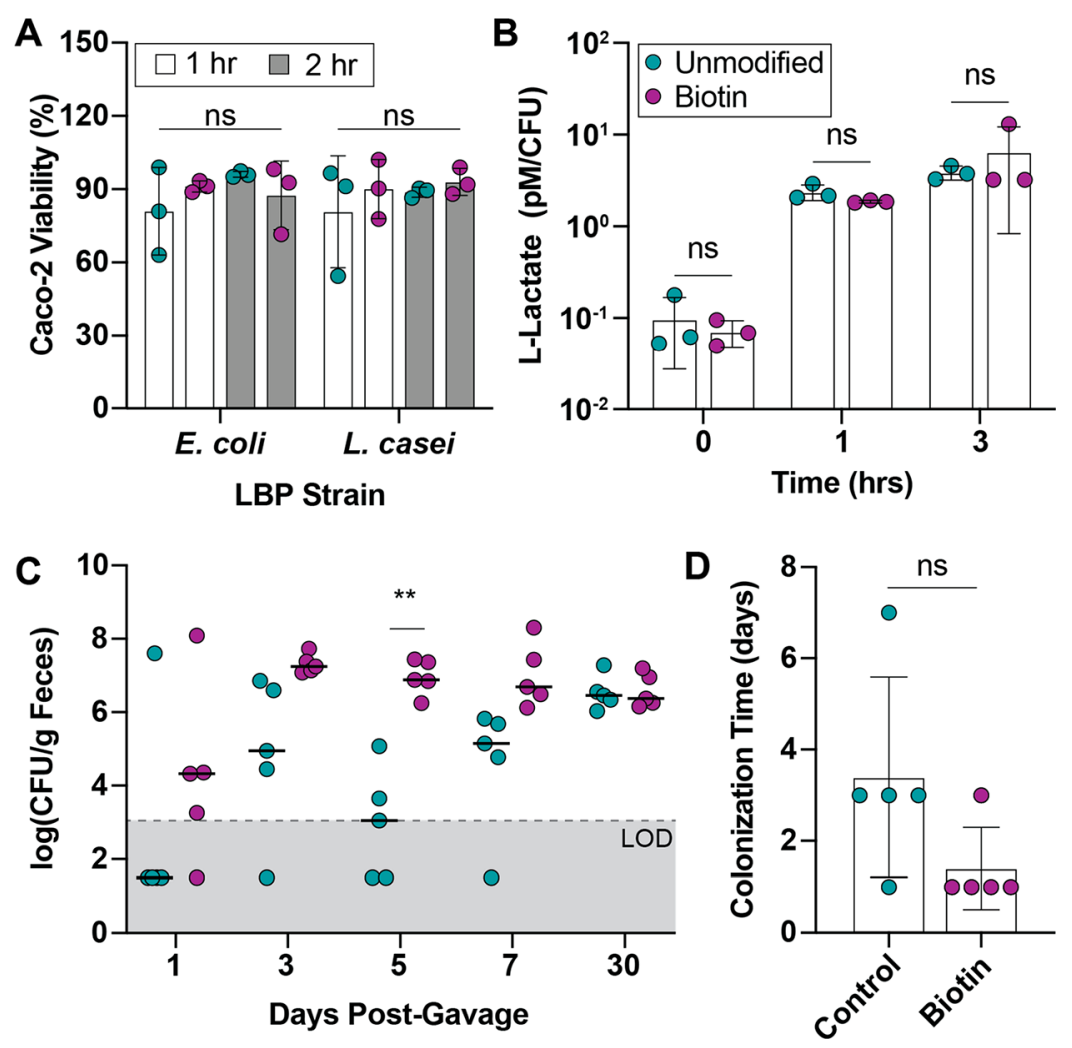

Figure 6. Surface biotinylation does not impede measures of LBP efficacy. (A) Viability of Caco-2 cells following incubation with unmodified (green) or biotinylated (pink) Escherichia coli Nissle 1917 or Lactobacillus casei for 1 (white) or 2 (gray) h, measured using an MTT assay ( $n=3$, bars represent standard deviation). (B) L-Lactate production in picomolar (pM) units from L. casei, normalized per colony forming unit (CFU) of bacteria in MRS media ( $n=3$, bars represent standard deviation). (C) Eight-week-old female BALB/c mice were treated with $10^{8} \mathrm{CFU}$ of unmodified or biotinylated $\mathrm{EcN}$, and fecal pellets were collected at indicated time points. Abundance of EcN was assessed by homogenizing fecal samples, plating on selective agar plates, and enumerating viable CFU $(n=5$, bars represent median, limit of detection $(\mathrm{LOD})=3$, values below LOD are shown as LOD/2). (D) Rate of EcN colonization, defined as the day at which detectable EcN was present in the feces of individual mice $(n=5$, bars represent standard deviation) (significance assessed using a two-way ANOVA with Sidak's post hoc test for multiple comparisons with $\alpha=0.05$ in $\mathrm{A}-\mathrm{C}$ or Log-rank Mantel-Cox test in $\mathrm{D}, * * p<0.01$, ns $=$ not significant).

Attachment has been shown to increase with residence time (i.e., the contact time between the microbe and its target) due to increased collisions with the attachment surface. ${ }^{34}$ Understanding the residence time required to enable sufficient attachment of LBPs to the GI tract is important for rationally designing oral delivery systems, as attachment is a critical step in the colonization of LBPs. ${ }^{35}$ For this study, E. coli DH5 $\alpha$ was incubated at a constant concentration $(\mathrm{OD}=0.25)$ for varying lengths of time ( $5 \mathrm{~min}$ to $24 \mathrm{~h}$ ) on a streptavidin-coated wellplate at $4{ }^{\circ} \mathrm{C}$ to limit growth and viability loss. At each time point, the well-plate was washed, and LBP attachment was quantified using fluorescence microscopy (Figure 4A and B, Figure S3B). Results are consistent with previously published studies, ${ }^{34,36}$ demonstrating that LBP attachment increases with residence time until saturation is reached, which occurs after approximately $2 \mathrm{~h}$. We observed modest decreases in the attachment of biotinylated LBPs after saturation was reached, likely reflecting loss of LBP viability following long-term incubation in PBS. Compared to optimizing the LBP concentration alone, we were able to nearly double the attachment of biotinylated LBPs by extending the residence time $(\sim 2100$ bacteria per frame in Figure $3 \mathrm{~B}$ vs $\sim 3800$ in Figure 4A).

2.3. Effects of Surface-Modifications on LBP Behavior. Current clinical use of LBPs, including fecal microbiota transplants $^{37}$ and donor-derived spore-based therapeutics, ${ }^{38}$ relies on defined processing steps that attempt to maximize viability and are compatible with cryopreservation. Therefore, we characterized the effect of surface modification on the growth, viability, and cryopreservation of LBPs. Biotinylation did not affect the growth of any LBP strain tested during an 8$\mathrm{h}$ period (Figure 5A), nor did biotinylation significantly alter LBP viability, measured as CFUs (Figure 5B). Clinically, preservation of LBP formulations is essential for their practicality, as they can rarely be used immediately upon preparation and may require transport between manufacturing facilities and clinics. As such, $\mathrm{EcN}$ was tested for its storage under common cryopreservation conditions (25\% glycerol, $-80{ }^{\circ} \mathrm{C}$ ). We selected $\mathrm{EcN}$ for this particular study, as it is one of the most clinically advanced LBPs. ${ }^{39}$ Surface modification did not significantly alter LBP viability for up to 5 months of storage (Figure 5C), and the functionality of surface modification proved to be compatible with cold storage, as streptavidin binding was preserved at each time point tested (Figure 5D and Figure S9). This presents a key advantage for this modification platform, as it improves the potential that LBP formulations can be prepared and modified at scale, prior to quality control, packaging, and storage processing steps. Additionally, the ability to modify LBPs prior to storage and shipping may allow for an off-the-shelf therapeutic that alleviates the need to conduct postpreservation modifications 
of the LBP at the point-of-care, which can be burdensome for patients, clinics, or hospitals. ${ }^{40}$

The ability to effectively access and use nutrient sources (metabolism) in order to survive and proliferate within the intestinal tract (colonization) without significantly influencing the health of their human host (mammalian toxicity) is essential for LBP therapeutic efficacy. While we confirmed that biotinylation does not inhibit LBP growth or viability, it is not clear if surface modifications alter these more complex bacterial functions. To determine the influence of surface modifications on these parameters, we first evaluated the viability of mammalian cells after exposure to the candidate LBPs EcN and $L$. casei using an MTT assay. LBPs were incubated on Caco-2 cells, a colorectal cancer cell line commonly used as a model of the intestinal epithelium, at concentrations ranging from $10^{6}$ to $10^{8} \mathrm{CFU} /$ well for up to $2 \mathrm{~h}$. We found no significant toxicity against mammalian cells for either the LBP strain following biotinylation at $10^{6}$ (Figure S10A) or $10^{7}$ $\mathrm{CFU} /$ well (Figure 6A). However, at high LBP concentrations $\left(10^{8} \mathrm{CFU} /\right.$ well), we found that $\mathrm{EcN}$ and L. casei contributed to the reduction of MTT bromide to formazan, ${ }^{41}$ resulting in signals above the positive control (Figure S10B) and decreasing the reliability of results. For this reason, we are limited to shorter time points for this MTT assay, as exponential LBP growth interferes with the MTT readout. While longer incubations would be required to make a definitive statement on the cytotoxicity of these LBP strains, biotin on the LBP surface rapidly dilutes during growth (Figure 2B), making these shorter incubations a more representative time window for comparing the toxicity of biotinylated and unmodified LBPs on Caco-2 cells.

Next, we tested the impact of biotinylation on the metabolism of an LBP strain. We selected L. casei, as it is known to produce a therapeutically active compound during growth that can be reliably quantified. $L$. casei produces lactic acid through the fermentation of glucose, which has been shown to mediate diverse disease states, including NSAIDinduced small intestine injury, ${ }^{42}$ diabetes complications, ${ }^{43}$ and pathogen infections. ${ }^{44}$ As such, it is essential that surface modifications of $L$. casei do not interfere with lactic acid secretion. We measured lactic acid production under two conditions: during growth in MRS media and during fermentation in minimal media supplemented with glucose to inhibit growth and ensure that biotin dilution did not influence results (Figure S11A). We found that biotinylation does not significantly alter lactic acid production under either growth condition (Figure 6B and Figure S11B).

We recently demonstrated that the attachment of mucusbinding, streptavidin-conjugated antibodies to biotinylated LBPs significantly improves their short-term adhesion in the GI tract, enabling them to quickly establish an intestinal niche and improving their pharmacokinetics. ${ }^{16}$ While targeting specific receptors in the GI tract appears to improve colonization, little is known about the influence of surface modifications themselves on the interactions between LBPs and the GI environment. Indeed, alternative approaches to modifying LBP surfaces, such as encapsulation, can physically impede LBP growth or interactions with the GI environment. Therefore, we were interested in analyzing the effect of our base platform, biotinylated LBPs, on the growth and colonization of an LBP in the murine GI tract. To ensure reliable detection of the LBP throughout the study, we dosed animals with $\mathrm{EcN}$, which harbors a kanamycin-resistance plasmid that is stable for long periods of time in vivo. ${ }^{45}$ To assess the colonization of $\mathrm{EcN}$, we treated female BALB/c mice with streptomycin and introduced either biotinylated or unmodified $\mathrm{EcN}$ via oral gavage. At indicated time points, we collected and homogenized fecal pellets as a proxy for the intestinal LBP abundance, which we have previously shown is an accurate approximation in this mouse model. ${ }^{16}$

The results show that colonization of the modified LBP is noninferior to the unmodified control (Figure 6C). For the first $72 \mathrm{~h}$, there are no significant differences between the groups, and during the full 30-day window that we collected fecal pellets, the two groups demonstrated significant differences only at Day 5. Additionally, there is no significant difference between the rate of colonization, measured as the number of days for viable $\mathrm{EcN}$ to appear in the feces of mice, between the groups (Figure 6D). For further evidence of the noninferiority of surface modified LBPs, we found that both biotinylated and unmodified LBPs stably colonized the murine GI tract at equivalent abundances out to 30 -days postgavage, as shown in Figure 6C. We further analyzed these results ex vivo by applying unmodified or biotinylated $E$. coli to the luminal side of pig intestine. We quantified the loss of LBP attachment to the intestinal lumen during consecutive washes using an In Vivo Imaging System (IVIS) (Figures S12A and S12B). Consistent with our in vivo findings, we found that biotinylated LBPs are noninferior to the unmodified control, and there was no significant difference in LBP attachment to the ex vivo intestine, measured using IVIS or plating on selective agar plates (Figures S12C and S12D). Therefore, modifying the LBP surface with a modular handle such as biotin does not significantly impede LBP attachment or colonization in the intestinal tract.

\section{CONCLUSION}

Modifications to the LBP surface are a promising method to alter their interactions with the human host and improve therapeutic efficacy. However, their use as an oral delivery strategy for LBPs remains poorly characterized. In this work, we analyzed a surface modification platform that bioconjugates biotin to the LBP surface, which can then act as a modular handle for the attachment of secondary moieties. Previously, we have shown that this platform is functional in vivo and that modification of the LBP surface with mucus-binding antibodies improves LBP colonization and pharmacokinetics. Herein, we expanded on this work by demonstrating that bioconjugation of biotin enables modification with any streptavidin-conjugated moiety, including antibodies, polymers, and nanoparticles. These modifications can potentially be used for diverse delivery applications, including hitchhiking of essential nutrients to the LBP surface to provide a competitive growth advantage in the GI tract, shielding of immunogenic antigens on the LBP surface using inert polymers, or targeting sites of disease with antibodies directed against wound targets.

Additionally, we have analyzed conditions influencing the oral delivery of LBPs, including the effect of LBP parameters (growth and biotin dilution, concentration, contact time) on the success of surface modifications as a delivery strategy, as well as the effect of the platform on measures of LBP efficacy and clinical translation (viability, toxicity, metabolism, colonization, and storage). Specifically, we used our base platform of biotinylated LBPs to analyze attachment to the target protein streptavidin. Using this approach, we found that LBP attachment is inhibited at high concentrations and 
saturates at extended contact times. While additional work is needed to correlate these findings to the in vivo environment, knowledge on the physical parameters influencing target attachment of modified LBPs can be used as a starting point to inform their oral delivery and in vivo colonization. For example, understanding the kinetics of LBP target binding could potentially motivate the selection of a delivery system that controls the spatiotemporal concentration of LBPs in the GI tract. Additionally, these findings can be used to optimize secondary ligand presentation in future work. We additionally found that LBP growth dilutes the concentration of targeting ligands on the LBP surface, inhibiting their attachment to target proteins and confirming that the LBP is reverted to premodification levels of attachment. This is an important feature of this platform, as it reduces safety concerns associated with long-term or permanent changes to the LBP. Future work will expand these findings to additional LBP strains, as these attachment optimums and growth conditions were investigated for a single LBP strain with a set surface concentration of biotin.

Finally, this work further demonstrated that NHS-esterbased bioconjugation does not significantly impede critical parameters known to influence LBP efficacy, including their growth, viability, metabolite secretion, and in vivo colonization. This latter demonstration is relevant to the use of this strategy as a delivery method to improve colonization, as alternative surface modifications, such as polymer encapsulation, may inhibit LBP growth or attachment. We further confirmed that our biotinylation platform does not impede LBP attachment to the GI lumen in an ex vivo porcine intestine model. Importantly, we have shown that surface modified LBPs can be stored for up to 5 months without influencing their viability or target binding, using clinically relevant storage conditions. While this work is limited in its scope due to the small number of LBP strains rigorously tested, as well as the variability between LBP strains and their bioconjugation efficiencies, it provides a foundation of support for bioconjugation-based surface modifications and establishes both key considerations for designing oral LBP delivery systems, as well as the experimental approaches for evaluating them.

\section{MATERIALS AND METHODS}

4.1. Cell Lines and Culture. Lactobacillus casei (ATCC 393) and Bacillus coagulans (ATCC 7050) were purchased from ATCC. Escherichia coli DH5 $\alpha$ was purchased transformed with either a pBS-ldhGFP plasmid conferring GFP-expression and ampicillin resistance (selection at $100 \mu \mathrm{g} / \mathrm{mL}$ ), a gift from Michela Lizier (Addgene plasmid \#27170; http://n2t.net/addgene:27170; RRID:Addgene_27170), or a pLEM415-ldhL-mRFP1 plasmid conferring RFP-expression and ampicillin resistance (selection at $100 \mu \mathrm{g} / \mathrm{mL}$ ), a gift from Sujin Bao (Addgene plasmid \# 99842; http://n2t.net/ addgene:99842; RRID:Addgene_99842). ${ }^{46-48}$ Escherichia coli Nissle 1917 was a gift from Nathan Crook and came transformed with a plasmid conferring kanamycin resistance (selection at $50 \mu \mathrm{g} / \mathrm{mL}){ }^{45}$ Glycerol stocks of all bacterial strains were prepared from overnight cultures, diluted $1: 1$ in $50 \%$ sterile glycerol. $L$. casei was grown in MRS media at $37{ }^{\circ} \mathrm{C}$ under static conditions, while B. coagulans (grown in Nutrient Broth) and E. coli strains (grown in Luria Broth) were grown at $37^{\circ} \mathrm{C}$ in a shaking incubator $(200 \mathrm{rpm})$. All bacterial cultures were inoculated from glycerol stocks at least $12 \mathrm{~h}$ before use in a study in media supplemented with appropriate concentrations of antibiotics. Caco-2 cells were purchased from the University of North Carolina at Chapel Hill Tissue Culture Facility and cultured in phenol-free Dulbecco's Modified Eagle's Medium (DMEM, Gibco) supplemented with $20 \%$ Fetal Bovine Serum (FBS) and 1\% penicillin-streptomycin.
4.2. Biotinylation of Bacteria and Secondary Moiety Binding. Bacteria cultures were grown overnight and biotinylated as previously described. ${ }^{16}$ Briefly, bacteria were harvested via centrifugation, washed twice in ice-cold PBS, diluted to an optical density measured at $600 \mathrm{~nm}$ (OD600) of 1.0, and reacted for $20 \mathrm{~min}$ on ice at a concentration of $1 \mathrm{mg} / \mathrm{mL}$ of $N$-hydroxysulfosuccinimide functionalized biotin (EZ-Link Sulfo-NHS Biotin; ThermoFisher). For the reactant concentration study (Figure S8), E. coli DH5 $\alpha$ was incubated with sulfo-NHS-biotin at concentrations ranging between 0.1 and $2.0 \mathrm{mg} / \mathrm{mL}$ in an OD $=1.0$ solution. Samples were washed $2 \times$ with PBS via centrifugation at $4,000 \mathrm{rpm}$ for $10 \mathrm{~min}$. $10 \mu \mathrm{L}$ of fluorescent streptavidin probe (Streptavidin Alexa Fluor 594 conjugate, Invitrogen) was mixed with $100 \mu \mathrm{L}$ of bacteria, washed as described above, and imaged using fluorescence microscopy (Revolve, Echo). Fluorescence intensity was measured using a microplate reader (Synergy H1, BioTek). For antibody attachment, a fluorescent IgG antibody (Goat Anti-Mouse IgG Alexa Fluor 647 Conjugate, Abcam) was conjugated to streptavidin using a kit, according to the manufacturer's instructions (Streptavidin Conjugation Kit, Abcam). Antibody was added to cultures of E. coli DH5 $\alpha$ at a concentration of $20 \mu \mathrm{g} / \mathrm{mL}$, briefly vortexed, washed as described above, and imaged using fluorescence microscopy. Similarly, streptavidin-conjugated nanoparticles (FluoSpheres StreptavidinLabeled Microspheres $0.04 \mu \mathrm{m}$, Life Technologies) were incubated at a dilution of 1:100 and processed in the same manner as the streptavidin-conjugated antibody. Finally, streptavidin-conjugated polymer (Streptavidin Peroxidase Polymer, Fisher Scientific) was incubated at a dilution of 1:100,000, vortexed briefly, and immediately washed $3 \times$ in PBS. On the final wash, samples were resuspended in $100 \mu \mathrm{L}$ of TMB ELISA substrate and incubated for $30 \mathrm{~min}$. The reaction was stopped with $100 \mu \mathrm{L}$ of $0.6 \mathrm{~N} \mathrm{H}_{2} \mathrm{SO}_{4}$, and sample absorbance was read at $450 \mathrm{~nm}$.

4.3. Biotin Dilution during Growth. E. coli DH5 $\alpha$ cultures were grown overnight and biotinylated as described above. Following washes, E. coli was diluted to an OD600 of $\sim 0.2$ and transferred to an incubator at $37{ }^{\circ} \mathrm{C}$. At each time point, samples were removed and used to quantify the surface biotin concentration, attachment to a streptavidin-coated plate, and bacterial concentration. A fluorescent streptavidin probe was used to calculate surface biotin concentration; the probe was incubated with samples as described above, and the number of streptavidin molecules was determined using a standard curve from the fluorescence intensity on a microplate reader (Synergy $\mathrm{H} 1$, BioTek). Attachment was determined by incubating samples on a streptavidin-coated plate (Pierce Streptavidin Coated High Binding Capacity Plate; Life Technologies) for 20 min while shaking at room temperature. Fluorescence intensity was measured prior to and following washes to remove unbound bacteria using a microplate reader. Images were captured on the bottom of the well plate (Echo; Revolve). Bacterial concentration was determined by plating samples on selective agar plates and enumerating colony forming units (CFU).

4.4. Attachment Studies. E. coli DH5 $\alpha$ was grown and biotinylated as previously described. Cultures were diluted to indicated OD600 and incubated on a streptavidin-coated well-plate for $20 \mathrm{~min}$ at room temperature, shaking on a microplate shaker. For the contact time study, samples were incubated at $4{ }^{\circ} \mathrm{C}$ for indicated time points under static conditions. For all attachment studies, wells were washed $4 \times$ with PBS to remove unbound bacteria. Fluorescence intensity was quantified on a microplate reader prior to and following washing (Synergy H1, BioTek), and three images at unique positions on the well floor were taken for each replicate (Revolve, Echo). For the growth of attached bacteria at varying concentrations, the well medium was replaced with fresh LB broth (supplemented with 100 $\mu \mathrm{g} / \mathrm{mL}$ ampicillin), and the microplate was transferred to an incubator at $37^{\circ} \mathrm{C}$. At 1-h increments, the well-plate was removed and washed $4 \times$ as described above, and images were taken of the well-plate floor for quantification. Image analysis was conducted using Particle Counting in ImageJ.

4.5. Viability, Growth, and Storage. LBPs were biotinylated as previously described. For viability assessment, samples were taken immediately prior to and following biotinylation, serially diluted in 
PBS, plated on selective agar plates, and enumerated for viable CFUs. Samples were then diluted 1:100 in fresh medium in triplicate, added to a 96-well-plate, and sealed (Breathe-Easy Sealing Membrane, Sigma). Growth curves were measured in a microplate reader (Synergy H1, BioTek) at $37{ }^{\circ} \mathrm{C}$ for $8 \mathrm{~h}$, reading absorbance at 600 $\mathrm{nm}$ every $10 \mathrm{~min}$. For storage studies, LBPs were diluted 1:1 in 50\% sterile glycerol in deionized water and frozen at $-80{ }^{\circ} \mathrm{C}$. At indicated time points, vials were thawed at room temperature, and CFUs were enumerated.

4.6. Mammalian Viability. Caco-2 cells were seeded in tissue culture treated 96 -well-plates $48 \mathrm{~h}$ before use at 10,000 cells/well. $L$. casei and $\mathrm{EcN}$ cultures were grown and biotinylated as described above and diluted to an OD of $0.8\left(\sim 10^{9} \mathrm{CFU} / \mathrm{mL}\right)$. Ten-fold dilutions were conducted in phenol-free DMEM to achieve a range of concentrations from $10^{7}-10^{9} \mathrm{CFU} / \mathrm{mL}$, and $100 \mu \mathrm{L}$ was added to Caco-2 wells. Cells were incubated for 1 or $2 \mathrm{~h}$, and an MTT assay was conducted according to the manufacturer's instructions (Vybrant MTT Cell Proliferation Assay Kit, Invitrogen), using DMSO to solubilize formazan in the final step. The average of a triplicate of untreated controls was used as the $100 \%$ viability reference point, while the average of a triplicate of $1 \%$ Triton-X-treated cells was used as the $0 \%$ viability reference point. Viability was calculated assuming a linear relationship.

4.7. Lactic Acid Secretion. L. casei was cultured and biotinylated as previously described. Cultures were collected via centrifugation at 4,000 rpm for $5 \mathrm{~min}$, resuspended in MRS media or M9 minimal media ( $5 \times$ M9 minimal Salts, BD Difco) supplemented with $0.4 \%$ glucose, and then diluted to an OD600 of 0.5. Samples were removed at $t=0$ and placed on ice, and cultures were transferred to $37^{\circ} \mathrm{C}$ in a static incubator. At indicated time points, samples were removed, bacteria were pelleted, and lactate concentration was assessed according to the manufacturer's instructions with the supernatant (L-Lactate Assay Kit, BioAssay Systems). L. casei concentration was quantified via plating on MRS agar plates and enumerating viable CFUs.

4.8. In Vivo Colonization of Modified Bacteria. Animal studies were conducted in accordance with and approved by the Institutional Animal Care and Use Committee (IACUC) of The University of North Carolina at Chapel Hill. Eight-week old female BALB/c mice were purchased from Charles River and acclimated for at least $72 \mathrm{~h}$ prior to use. Mice were placed on a controlled diet (OpenStandard Diet; Research Diets) for 7 days prior to the start of any studies. Streptomycin was given ad lib in the drinking water for $24 \mathrm{~h}(5 \mathrm{~g} / \mathrm{L})$, followed by an 18 -h wash-out period. Mice were gavaged with $100 \mu \mathrm{L}$ of $10^{9} \mathrm{CFU} / \mathrm{mL}$ of $\mathrm{EcN}$ in sterile saline $\left(10^{8} \mathrm{CFU}\right.$ total) following biotinylation, as described above, with flexible 20-gauge gavage needles $(30 \mathrm{~mm}$, Instech). Feces were collected from mice as previously described, ${ }^{16}$ homogenized in PBS, serially diluted, and plated on kanamycin selective LB agar plates $(50 \mu \mathrm{g} / \mathrm{mL})$.

4.9. Statistical Analysis. Statistical analyses were conducted using Graphpad Prism version 8.4.3 for macOS.

\section{ASSOCIATED CONTENT}

\section{(s) Supporting Information}

The Supporting Information is available free of charge at https://pubs.acs.org/doi/10.1021/acsbiomaterials.0c01405.

Supplemental methods for determination of primary amines using Kaiser test and evaluation of LBP attachment in ex vivo porcine intestine model; Figure $S 1$, extent of biotinylation and primary amine density varies between LBP strains; Figure S2, calibration curve of Alexa Fluor 594 fluorescent streptavidin probe; Figure S3, correlation between attached E. coli DH5 $\alpha$ and fluorescent signal; Figure S4, correlation between fluorescent signal and viable E. coli $\mathrm{DH} 5 \alpha$; Figure S5, attachment of unmodified LBPs increases with concentration; Figure S6, attachment maximum varies between study conditions but attachment efficiency of biotiny- lated LBPs decreases with concentration; Figure S7, biotinylated LBPs detach during growth while retaining attachment advantages over unmodified LBPs; Figure S8, extent of biotinylation with varying reactant concentrations and effect on attachment; Figure S9, streptavidin attachment following storage at $-80{ }^{\circ} \mathrm{C}$; Figure S10, biotinylation does not significantly alter toxicity of LBPs; Figure S11, biotinylation does not alter LBP metabolism, regardless of growth condition; and Figure S12, attachment of biotinylated or unmodified LBPs to ex vivo pig intestine (PDF)

\section{AUTHOR INFORMATION}

\section{Corresponding Author}

Aaron C. Anselmo - Division of Pharmacoengineering and Molecular Pharmaceutics, Eshelman School of Pharmacy, University of North Carolina at Chapel Hill, Chapel Hill, North Carolina 27599, United States; 1 orcid.org/00000003-3290-7857; Email: aanselmo@email.unc.edu

\section{Author}

Ava M. Vargason - Division of Pharmacoengineering and Molecular Pharmaceutics, Eshelman School of Pharmacy, University of North Carolina at Chapel Hill, Chapel Hill, North Carolina 27599, United States

Complete contact information is available at:

https://pubs.acs.org/10.1021/acsbiomaterials.0c01405

\section{Author Contributions}

A.C.A. and A.M.V. conceived of the presented idea. A.M.V. planned and carried out experiments, created visuals, conducted statistical analyses, and wrote the manuscript with support from A.C.A. A.C.A. supervised the experimental planning and findings of this work. All authors have given approval to the final version of the manuscript.

\section{Notes}

The content is solely the responsibility of the authors and does not necessarily represent the official views of the National Institutes of Health.

The authors declare the following competing financial interest(s): A.M.V. and A.C.A. are listed as inventors on a patent application filed by The University of North Carolina at Chapel Hill on parts of the work presented here.

\section{ACKNOWLEDGMENTS}

A.M.V. was supported by the National Science Foundation Graduate Research Fellowship under Grant No. DGE1650116. Research reported in this publication was supported by the National Institute of General Medical Sciences of the National Institutes of Health under Award Number R35GM137898.

\section{REFERENCES}

(1) Singh, R. K.; Chang, H. W.; Yan, D.; Lee, K. M.; Ucmak, D.; Wong, K.; Abrouk, M.; Farahnik, B.; Nakamura, M.; Zhu, T. H.; Bhutani, T.; Liao, W. Influence of diet on the gut microbiome and implications for human health. J. Transl. Med. 2017, 15 (1), 73.

(2) Clayton, T. A.; Baker, D.; Lindon, J. C.; Everett, J. R.; Nicholson, J. K. Pharmacometabonomic identification of a significant hostmicrobiome metabolic interaction affecting human drug metabolism. Proc. Natl. Acad. Sci. U. S. A. 2009, 106 (34), 14728-33.

(3) Donohoe, D. R.; Garge, N.; Zhang, X.; Sun, W.; O'Connell, T. M.; Bunger, M. K.; Bultman, S. J. The microbiome and butyrate 
regulate energy metabolism and autophagy in the mammalian colon. Cell Metab. 2011, 13 (5), 517-26.

(4) Goossens, H.; Ferech, M.; Vander Stichele, R.; Elseviers, M. Outpatient antibiotic use in Europe and association with resistance: a cross-national database study. Lancet 2005, 365 (9459), 579-587.

(5) French, G. L. The continuing crisis in antibiotic resistance. Int. J. Antimicrob. Agents 2010, 36 (3), S3-S7.

(6) Vargason, A. M.; Anselmo, A. C. Clinical translation of microbebased therapies: Current clinical landscape and preclinical outlook. Bioeng Transl Med. 2018, 3 (2), 124-137.

(7) Pinero-Lambea, C.; Ruano-Gallego, D.; Fernandez, L. A. Engineered bacteria as therapeutic agents. Curr. Opin. Biotechnol. 2015, 35, 94-102.

(8) Isabella, V. M.; Ha, B. N.; Castillo, M. J.; Lubkowicz, D. J.; Rowe, S. E.; Millet, Y. A.; Anderson, C. L.; Li, N.; Fisher, A. B.; West, K. A.; Reeder, P. J.; Momin, M. M.; Bergeron, C. G.; Guilmain, S. E.; Miller, P. F.; Kurtz, C. B.; Falb, D. Development of a synthetic live bacterial therapeutic for the human metabolic disease phenylketonuria. Nat. Biotechnol. 2018, 36 (9), 857-864.

(9) Prakash, S.; Jones, M. L. Artificial Cell Therapy: New Strategies for the Therapeutic Delivery of Live Bacteria. J. Biomed. Biotechnol. 2005, 2005 (1), 44-56.

(10) Cook, M. T.; Tzortzis, G.; Charalampopoulos, D.; Khutoryanskiy, V. V. Microencapsulation of probiotics for gastrointestinal delivery. J. Controlled Release 2012, 162 (1), 56-67.

(11) Anselmo, A. C.; McHugh, K. J.; Webster, J.; Langer, R.; Jaklenec, A. Layer-by-Layer Encapsulation of Probiotics for Delivery to the Microbiome. Adv. Mater. 2016, 28 (43), 9486-9490.

(12) Park, B.-W.; Zhuang, J.; Yasa, O.; Sitti, M. Multifunctional Bacteria-Driven Microswimmers for Targeted Active Drug Delivery. ACS Nano 2017, 11 (9), 8910-8923.

(13) Jonas, A. M.; Glinel, K.; Behrens, A.; Anselmo, A. C.; Langer, R. S.; Jaklenec, A. Controlling the Growth of Staphylococcus epidermidis by Layer-By-Layer Encapsulation. ACS Appl. Mater. Interfaces 2018, 10 (19), 16250-16259.

(14) Priya, A. J.; Vijayalakshmi, S.; Raichur, A. M. Enhanced survival of probiotic Lactobacillus acidophilus by encapsulation with nanostructured polyelectrolyte layers through layer-by-layer approach. J. Agric. Food Chem. 2011, 59 (21), 11838-11845.

(15) Cook, M. T.; Tzortzis, G.; Khutoryanskiy, V. V.; Charalampopoulos, D. Layer-by-layer coating of alginate matrices with chitosan-alginate for the improved survival and targeted delivery of probiotic bacteria after oral administration. J. Mater. Chem. B 2013, 1 (1), 52-60.

(16) Vargason, A. M.; Santhosh, S.; Anselmo, A. C. Surface Modifications for Improved Delivery and Function of Therapeutic Bacteria. Small 2020, 16 (25), 2001705.

(17) Cao, Z.; Cheng, S.; Wang, X.; Pang, Y.; Liu, J. Camouflaging bacteria by wrapping with cell membranes. Nat. Commun. 2019, 10 (1), 3452 .

(18) Fernandes, R.; Zuniga, M.; Sassine, F. R.; Karakoy, M.; Gracias, D. H. Enabling Cargo-Carrying Bacteria via Surface Attachment and Triggered Release. Small 2011, 7 (5), 588-592.

(19) Kozlovskaya, V.; Harbaugh, S.; Drachuk, I.; Shchepelina, O.; Kelley-Loughnane, N.; Stone, M.; Tsukruk, V. V. Hydrogen-bonded LbL shells for living cell surface engineering. Soft Matter 2011, 7 (6), 2364-2372.

(20) Wang, X.; Cao, Z.; Zhang, M.; Meng, L.; Ming, Z.; Liu, J. Bioinspired oral delivery of gut microbiota by self-coating with biofilms. Science Advances 2020, 6 (26), eabb1952.

(21) Lahav-Mankovski, N.; Prasad, P. K.; Oppenheimer-Low, N.; Raviv, G.; Dadosh, T.; Unger, T.; Salame, T. M.; Motiei, L.; Margulies, D. Decorating bacteria with self-assembled synthetic receptors. Nat. Commun. 2020, 11 (1), 1299.

(22) Ho, C. L.; Tan, H. Q.; Chua, K. J.; Kang, A.; Lim, K. H.; Ling, K. L.; Yew, W. S.; Lee, Y. S.; Thiery, J. P.; Chang, M. W. Engineered commensal microbes for diet-mediated colorectal-cancer chemoprevention. Nature Biomedical Engineering 2018, 2 (1), 27-37.
(23) Piñero-Lambea, C.; Bodelón, G.; Fernández-Periáñez, R.; Cuesta, A. M.; Álvarez-Vallina, L.; Fernández, L. Á. Programming Controlled Adhesion of E. coli to Target Surfaces, Cells, and Tumors with Synthetic Adhesins. ACS Synth. Biol. 2015, 4 (4), 463-473.

(24) Mays, Z. J. S.; Chappell, T. C.; Nair, N. U. Quantifying and Engineering Mucus Adhesion of Probiotics. ACS Synth. Biol. 2020, 9 (2), 356-367.

(25) Drachuk, I.; Shchepelina, O.; Lisunova, M.; Harbaugh, S.; Kelley-Loughnane, N.; Stone, M.; Tsukruk, V. V. pH-responsive layerby-layer nanoshells for direct regulation of cell activity. ACS Nano 2012, 6 (5), 4266-78.

(26) Carter, J. L.; Drachuk, I.; Harbaugh, S.; Kelley-Loughnane, N.; Stone, M.; Tsukruk, V. V. Truly nonionic polymer shells for the encapsulation of living cells. Macromol. Biosci. 2011, 11 (9), 1244-53.

(27) Gunsolus, I. L.; Hu, D.; Mihai, C.; Lohse, S. E.; Lee, C. S.; Torelli, M. D.; Hamers, R. J.; Murhpy, C. J.; Orr, G.; Haynes, C. L. Facile method to stain the bacterial cell surface for super-resolution fluorescence microscopy. Analyst 2014, 139 (12), 3174-8.

(28) Cox, J. S.; Smith, D. S.; Warren, L. A.; Ferris, F. G. Characterizing Heterogeneous Bacterial Surface Functional Groups Using Discrete Affinity Spectra for Proton Binding. Environ. Sci. Technol. 1999, 33 (24), 4514-4521.

(29) Hinds, K. D.; Kim, S. W. Effects of PEG conjugation on insulin properties. Adv. Drug Delivery Rev. 2002, 54 (4), 505-530.

(30) Kim, P.-H.; Kim, T.-i.; Yockman, J. W.; Kim, S. W.; Yun, C.-O. The effect of surface modification of adenovirus with an argininegrafted bioreducible polymer on transduction efficiency and immunogenicity in cancer gene therapy. Biomaterials 2010, 31 (7), $1865-1874$

(31) Akin, D.; Sturgis, J.; Ragheb, K.; Sherman, D.; Burkholder, K.; Robinson, J. P.; Bhunia, A. K.; Mohammed, S.; Bashir, R. Bacteriamediated delivery of nanoparticles and cargo into cells. Nat. Nanotechnol. 2007, 2 (7), 441-449.

(32) Chan, C. T.; Lee, J. W.; Cameron, D. E.; Bashor, C. J.; Collins, J. J. 'Deadman' and 'Passcode' microbial kill switches for bacterial containment. Nat. Chem. Biol. 2016, 12 (2), 82-6.

(33) Riglar, D. T.; Silver, P. A. Engineering bacteria for diagnostic and therapeutic applications. Nat. Rev. Microbiol. 2018, 16 (4), 214225.

(34) Fletcher, M. The effects of culture concentration and age, time, and temperature on bacterial attachment to polystyrene. Can. J. Microbiol. 1977, 23 (1), 1-6.

(35) Gonzalez-Rodriguez, I.; Ruiz, L.; Gueimonde, M.; Margolles, A.; Sanchez, B. Factors involved in the colonization and survival of bifidobacteria in the gastrointestinal tract. FEMS Microbiol. Lett. 2013, 340 (1), 1-10.

(36) Ludwicka, A.; Jansen, B.; Wadström, T.; Pulverer, G. Attachment of staphylococci to various synthetic polymers. Zentralbl. Bakteriol., Mikrobiol. Hyg., Abt. 1, Orig. A 1984, 256 (4), 479-489.

(37) Satokari, R.; Mattila, E.; Kainulainen, V.; Arkkila, P. Simple faecal preparation and efficacy of frozen inoculum in faecal microbiota transplantation for recurrent $\mathrm{C}$ lostridium difficile infection-an observational cohort study. Aliment. Pharmacol. Ther. 2015, 41 (1), $46-53$.

(38) Khanna, S.; Pardi, D. S.; Kelly, C. R.; Kraft, C. S.; Dhere, T.; Henn, M. R.; Lombardo, M. J.; Vulic, M.; Ohsumi, T.; Winkler, J.; Pindar, C.; McGovern, B. H.; Pomerantz, R. J.; Aunins, J. G.; Cook, D. N.; Hohmann, E. L. A Novel Microbiome Therapeutic Increases Gut Microbial Diversity and Prevents Recurrent Clostridium difficile Infection. J. Infect. Dis. 2016, 214 (2), 173-81.

(39) Kurtz, C. B.; Millet, Y. A.; Puurunen, M. K.; Perreault, M.; Charbonneau, M. R.; Isabella, V. M.; Kotula, J. W.; Antipov, E.; Dagon, Y.; Denney, W. S. An engineered E. coli Nissle improves hyperammonemia and survival in mice and shows dose-dependent exposure in healthy humans. Sci. Transl. Med. 2019, 11 (475), eaau7975.

(40) Coopman, K.; Medcalf, N. From production to patient: challenges and approaches for delivering cell therapies. In StemBook; 
Harvard Stem Cell Institute: Cambridge, MA, 2008; DOI: 10.3824/ stembook.1.97.1.

(41) Grela, E.; Kozlowska, J.; Grabowiecka, A. Current methodology of MTT assay in bacteria - A review. Acta Histochem. 2018, 120 (4), 303-311.

(42) Watanabe, T.; Nishio, H.; Tanigawa, T.; Yamagami, H.; Okazaki, H.; Watanabe, K.; Tominaga, K.; Fujiwara, Y.; Oshitani, N.; Asahara, T.; Nomoto, K.; Higuchi, K.; Takeuchi, K.; Arakawa, T. Probiotic Lactobacillus casei strain Shirota prevents indomethacininduced small intestinal injury: involvement of lactic acid. Am. J. Physiol Gastrointest Liver Physiol 2009, 297 (3), G506-13.

(43) Yadav, H.; Jain, S.; Sinha, P. R. Oral administration of dahi containing probiotic Lactobacillus acidophilus and Lactobacillus casei delayed the progression of streptozotocin-induced diabetes in rats. J. Dairy Res. 2008, 75 (2), 189-95.

(44) Hudault, S.; Lievin, V.; Bernet-Camard, M. F.; Servin, A. L. Antagonistic activity exerted in vitro and in vivo by Lactobacillus casei (strain GG) against Salmonella typhimurium C5 infection. Appl. Environ. Microbiol. 1997, 63 (2), 513-8.

(45) Crook, N.; Ferreiro, A.; Gasparrini, A. J.; Pesesky, M. W.; Gibson, M. K.; Wang, B.; Sun, X.; Condiotte, Z.; Dobrowolski, S.; Peterson, D.; Dantas, G. Adaptive Strategies of the Candidate Probiotic E. coli Nissle in the Mammalian Gut. Cell Host Microbe 2019, 25 (4), 499-512.e8.

(46) Lizier, M.; Sarra, P. G.; Cauda, R.; Lucchini, F. Comparison of expression vectors in Lactobacillus reuteri strains. FEMS Microbiol. Lett. 2010, 308 (1), 8-15.

(47) Lane, M. C.; Alteri, C. J.; Smith, S. N.; Mobley, H. L. Expression of flagella is coincident with uropathogenic Escherichia coli ascension to the upper urinary tract. Proc. Natl. Acad. Sci. U. S. A. 2007, 104 (42), 16669-74.

(48) Bao, S.; Zhu, L.; Zhuang, Q.; Wang, L.; Xu, P. X.; Itoh, K.; Holzman, I. R.; Lin, J. Distribution dynamics of recombinant Lactobacillus in the gastrointestinal tract of neonatal rats. PLoS One 2013, 8 (3), e60007. 